\title{
ChemComm
}

View Article Online

CORRECTION

View Journal | View Issue

Check for updates

Cite this: Chem. Commun., 2018, 54,7858

DOI: $10.1039 / \operatorname{cocc} 90292 \mathrm{e}$

rsc.li/chemcomm

\section{Correction: Development of glycosynthases with broad glycan specificity for the efficient glyco-remodeling of antibodies}

\author{
Sachin S. Shivatare, ${ }^{a}$ Lin-Ya Huang, ${ }^{a}$ Yi-Fang Zeng, ${ }^{a}$ Jung-Yu Liao, ${ }^{a}$ Tsai-Hong You, ${ }^{a}$ \\ Shi-Yun Wang, ${ }^{a}$ Ting Cheng, ${ }^{b}$ Chih-Wei Chiu, ${ }^{a}$ Ping Chao, ${ }^{a}$ Li-Tzu Chen, ${ }^{a}$ Tsung-I Tsai, ${ }^{c}$ \\ Chiu-Chen Huang, ${ }^{a}$ Chung-Yi Wu, ${ }^{* b}$ Nan-Horng Lin*a and Chi-Huey Wong ${ }^{\star b c}$
}

Correction for 'Development of glycosynthases with broad glycan specificity for the efficient glycoremodeling of antibodies' by Sachin S. Shivatare et al., Chem. Commun., 2018, 54, 6161-6164.

The authors regret that there was an error in Fig. 3 in the original manuscript. The value for the Fc $\gamma$ IIIA binding of Rtx-G16 in Fig. 3 was given as 5.4 but should be 33. The corrected version of Fig. 3 is presented below. There was also an error in the original caption. The last sentence in the caption referred to "maximal Fc $\gamma$ IIA binding". This should have read "maximal Fc $\gamma$ IIIA binding".

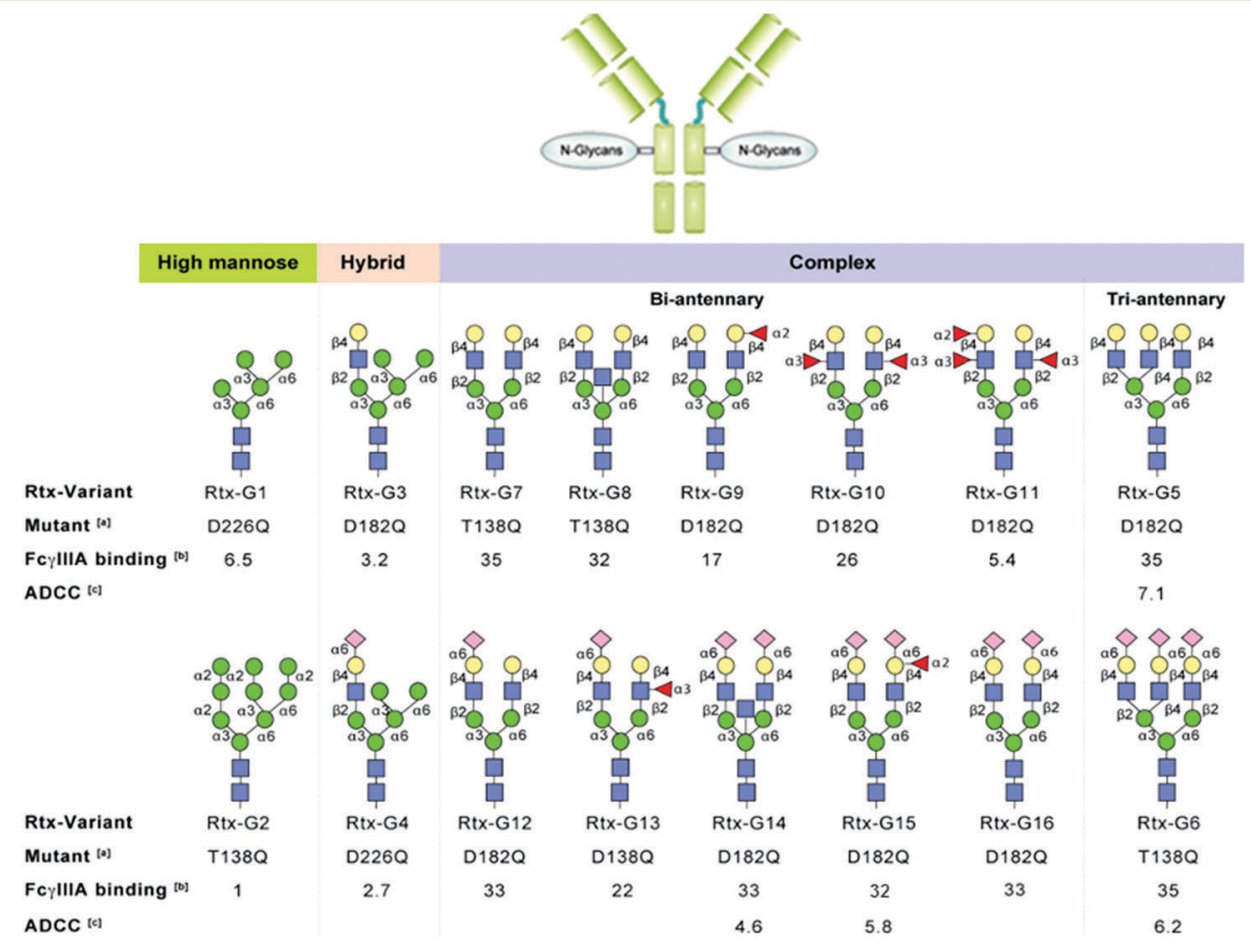

Fig. 3 Rtx-variants generated via Fc-glycosylation using Rtx-N as an acceptor and various glycan oxazolines as donors. (a) EndoS2 mutant required. (b) Binding between FcyRIIIA and Rtx-variants. Fold of enhancement of EC 50 compared to commercial Rtx. (c) ADCC activities of selected Rtx-variants. Fold of enhancement of $\mathrm{EC}_{50}$ compared to commercial Rtx. $\mathrm{EC}_{50}$ in $\mathrm{ng} \mathrm{\textrm {mL } ^ { - 1 }}$ refers to the concentration of an antibody that gives $50 \%$ of the maximal FcylllA binding or maximal cell killing.

The Royal Society of Chemistry apologises for these errors and any consequent inconvenience to authors and readers.

\footnotetext{
${ }^{a}$ CHO Pharma Inc., 18F, Building F, No. 3, Park Street, Nangang District, Taipei-11503, Taiwan. E-mail: nhlin@chopharma.com.tw

${ }^{b}$ Genomics Research Center, Academia Sinica, 128 Academia Road, Section 2, Nankang District, Taipei 115, Taiwan. E-mail: cyiwu@gate.sinica.edu.tw, chwong@gate.sinica.edu.tw

${ }^{c}$ Department of Chemistry, The Scripps Research Institute, 10550 North Torrey Pines Road, La Jolla, California 92037, USA
} 